\title{
Anabases
}

ANABASES Traditions et réceptions de l'Antiquité

$27 \mid 2018$

Varia

\section{Restituer les décors perdus de la maison de Neptune et Amphitrite : enquête, méthodes, résultats}

Hélène Eristov et Marie-Laure Maraval

\section{OpenEdition}

Journals

Édition électronique

URL : http://journals.openedition.org/anabases/6836

DOI : $10.4000 /$ anabases.6836

ISSN : 2256-9421

Éditeur

E.R.A.S.M.E.

\section{Édition imprimée}

Date de publication : 1 avril 2018

Pagination : 85-94

ISSN : 1774-4296

\section{Référence électronique}

Hélène Eristov et Marie-Laure Maraval, «Restituer les décors perdus de la maison de Neptune et Amphitrite : enquête, méthodes, résultats », Anabases [En ligne], 27 | 2018, mis en ligne le 01 avril 2020, consulté le 20 janvier 2021. URL : http://journals.openedition.org/anabases/6836 ; DOI : https:// doi.org/10.4000/anabases.6836 


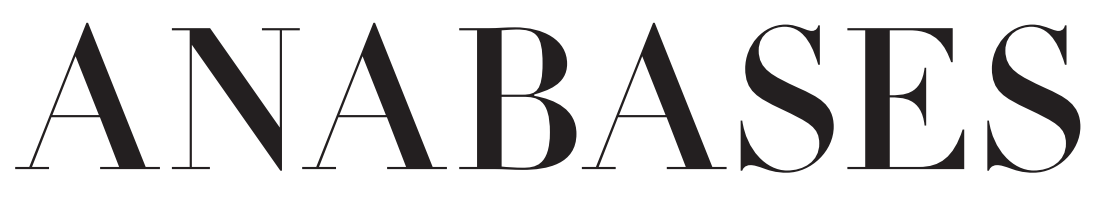

Traditions et Réceptions de l'Antiquité

$$
\begin{aligned}
& \text { No27 } \\
& 2018
\end{aligned}
$$

E.R.A.S.M.E.

Université Toulouse - Jean Jaurès 



\section{Sommaire}

$\mathrm{N}^{\circ} 27-2018$

\section{Historiographie et identités culturelles}

\section{Sébastien Cazalas}

“ et pour ce recite Valere... ». La place de l'Antiquité dans l'arsenal

intellectuel d'un grand prélat français du XVe siècle :

Jean Juvénal des Ursins (I388-I473) . . . . . . . . . . . . . . . . . . . II

Loic Marcou

La réception de l'Antiquité dans l'œuvre de la femme de lettres grecque

Rhéa Galanaki. . . . . . . . . . . . . . . . . . . . . .

\section{Traditions du patrimoine antique}

« Restituer Herculanum II. Des archives de fouilles aux restitutions 3D

Alexandra DARDENAY

Introduction : restituer l'espace domestique à Herculanum

grâce aux outils informatiques de reconstruction virtuelle ;

enjeux et problèmes. . . . . . . . . . . . . . . 4

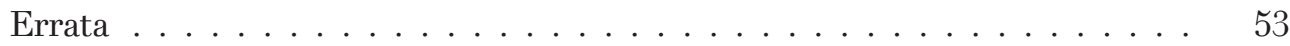

Carla Marotta

L’utilisation des données archivistiques dans l'enquête archéologique : nouvelles découvertes sur la maison d'Argos . . . . . . . . . . . . . . .

Emmanuelle Rosso

Restituer l'emplacement des statues dans les édifices d'Herculanum?

Problèmes de méthode . . . . . . . . . . . . . . . 7I 
Hélène Eristov, Marie-Laure Maraval

Restituer les décors perdus de la maison de Neptune et d'Amphitrite :

enquête, méthodes, résultats . . . . . . . . . . . . 9 9I

Maud Mulliez

Restauration numérique des peintures murales de la maison

de Neptune et Amphitrite à Herculanum : de l'expérimentation

matérielle à l'intégration des données dans un modèle 3D . . . . . . . Io3

\section{Archéologie des savoirs}

Nicolas SIRON

L'histoire de Philippidès d'Hérodote à Lucien.

Une incursion dans l'atelier du mythe . . . . . . . . . . . . . . . . Iog

Constantin Raios

Le coracin (xo@axîvos) du lac de Tibériade (F. Josèphe, B.J. III, 520) . . .

\section{Actualités et débats}

Claude Azıza

Antiquités parallèles (8). Le syndrome du sein droit . . . . . . . . . . . . I6I

Elisabeth DÉcultot

Winckelmann. Moderne Antike / Winckelmann. Modern Antiquity.

Exposition, Weimar, Neues Museum, 7 avril-2 juillet 20I7 . . . . . . . I67

\section{Lire, relire la bibliothèque des sciences de l'Antiquité}

Cyrielle LANDrea

Jérôme Carcopino et la mémoire perdue

de M. Valerius Messalla Corvinus (cos. 3г av. J.-C.) . . . . . . . . . . . . $\quad$ I77

Jérôme CARCopino

Notes biographiques sur M. Valerius Messala Corvinus . . . . . . . . . I I85

\section{L'atelier de l'histoire : chantiers historiographiques}

L'Atelier des doctorants (coordonné par Adeline Grand-Clément) (15)

Jonathan Pérez Mostazo

Cantabri aut vascones. La reception de la Antigüedad

en la cultura histórica vasca del siglo XIX . . . . . . . . . . . . . . . . . 209 


\section{Comptes rendus}

William H.F. Altman (éd.)

Brill's Companion to the Reception of Cicero (A. A. Raschieri). . . . . . . . 2 2I9

Anthony A. Barrett, Elaine Fantham, John C. Yardley

The Emperor Nero. A Guide to the Ancient Sources (É. Deniaux) . . . . . . 22I

Pascale Barthélémy et Violaine sébillotte cuchet (éd.)

Clio. Femmes, Genre, Histoire $\mathrm{n}^{\circ} 43$ : Citoyennetés (M. L. Napolitano) . . . 222

Frédéric Colin, Olivier Huck, Sylvie Vanseveren (éd.)

Interpretatio. Traduire l'altérité culturelle dans les civilisations

de l'Antiquité (Cl. Joncheray) . . . . . . . . . . . . . . . . . . . . 224

Angus Fletcher

Comic Democracies. From Ancient Athens

to the American Republic (A. de Crémoux). . . . . . . . . . . . . . . . . . 226

David Hernández de la Fuente

El despertar del alma. Dioniso y Ariadna: mito y misterio (A. Iriarte) . . . . 228

Ted Kaizer (éd.)

Religion, Society and Culture at Dura-Europos (P. G. Michelotto) . . . . . 229

Jacques Le Goff, Jean-Pierre Vernant

Dialogue sur l'histoire. Entretiens avec Emmanuel Laurentin (C. Lucci). . 23I

Justine Mac Connell, Edith Hall

Ancient Greek Myth in World Fiction since ig8g (A. de Crémoux) . . . . . . 233

Angelo Mazzocco, Marc Laureys (éd.)

ANew Sense of the Past. The Scholarship

of Biondo Flavio (I3g2-I463) (C. Lucci). . . . . . . . . . . . . . . . . . . . . . 234

Seth L. Schein

Homeric Epic and Its Reception. Interpretative Essays (S. Sistac) . . . . . . 236

Arbogast SснмiтT

Wie aufgeklärt ist die Vernunft der Aufklärung?

Eine Kritik aus aristotelischer Sicht (L. Calvié) . . . . . . . . . . . . . . . . 238

Richard Sorabu (éd.)

Aristotle Re-Interpreted. New Findings on Seven Hundred Years

of the Ancient Commentators (P. Butti de Lima) . . . . . . . . . . . . . . . 239

Laura SwiFT

Greek Tragedy. Themes and Contexts (G. Aujac) . . . . . . . . . . . . . . 24

Zara Martirosova TorLone

Vergil in Russia. National Identity and Classical Reception

(D. Millet-Gérard) . . . . . . . . . . . . . . . . . . 243 
Jürgen von UNGERN-STERNBERG

Les chers ennemis. Deutsche und französische

Altertumswissenschaftler in Rivalität und Zusammenarbeit (L. Calvié) . . 2444

Robert W. WALLACE

Reconstructing Damon: Music, Wisdom Teaching, and Politics

in Perikles'Athens (A. Brancacci) . . . . . . . . . . . . . . . . . . . . . 246

T. P. Wiseman

The Roman Audience : Classical Literature as Social History (C. Landrea) 249

Résumés . . . . . . . . . . . . . . . . . . . . . . . . . . 253

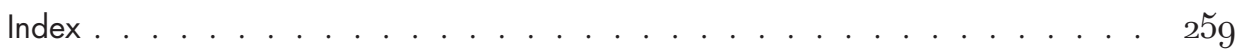




\section{Traditions}

\section{du patrimoine antique}

Restituer Herculanum II.

Des archives de fouilles aux restitutions 3D 



\section{Restituer les décors perdus de la maison de Neptune et Amphitrite : enquête, méthodes, résultats}

Hélène Eristov, Marie-Laure Maraval

'état des décors d'Herculanum présente des disparités avec ce que l'on observe à Pompéi, disparité qui tient à la chronologie des fouilles : commencées dès

- 1738 selon les méthodes des Bourbons, orientées vers le collectionnisme et marquées par le prélèvement systématique des “ morceaux » jugés dignes du musée royal, ces explorations reprennent massivement à partir des années 1930 et tendent alors à faire du site un musée à ciel ouvert où l'ensemble des trouvailles est laissé in situ. L'une et l'autre méthode ont abouti à des difficultés de lecture des décors en raison à la fois du déplacement des éléments figurés et du palissement ou de la quasi-disparition de pans entiers d'enduits peints. L'une des visées du programme VESUVIA est donc de replacer virtuellement les éléments prélevés dans leur contexte originel et de restituer autant que possible l'aspect des parois à l'aide de la documentation d'archives disponible'. D'emblée il faut noter que le décor pariétal romain présente certains avantages: son caractère relativement répétitif ainsi que le dispositif symétrique des parois permettent des extrapolations à partir d'éléments même fragmentaires ou ténus. Mais la méthodologie des fouilles et des restaurations d'Amedeo Maiuri introduit une difficulté : la notion d'in situ est très relative compte tenu du fait que les parois, fragilisées et par l'éruption et par les cunicoli des Bourbons, ont été reconstruites et leurs enduits déposés et replacés ; le doute méthodique s'impose donc.

1 Pour ce qui concerne l'histoire des fouilles et l'exploitation des archives, voir le dossier publié dans Anabases 26 (2017). 
Les méthodes de restitution mises en œuvre sont illustrées par deux cas de figure très différents: d'une part une petite pièce privée, semble-t-il, de toute documentation d'archives antérieure aux années 1970, mais au décor archéologiquement complet et auquel s'ajoutent deux prélèvements de l'époque des Bourbons; d'autre part le vaste atrium quasi-totalement dépouillé de ses décors, sans prélèvement repérable, mais où nous disposons de deux séries de fragments-clés et d'une photo d'archives qui a permis un ensemble de déductions.

\section{Le décor de la pièce 4}

Ce petit cubiculum (3,62 x 2,45 m) situé à gauche du tablinum ouvre à l'ouest directement sur l'atrium et à l'est, par une fenêtre haute, sur le nymphée.

La fouille de la pièce 4, alors numérotée 10, n'a laissé dans les archives de Maiuri $^{2}$ que la mention, datée du 9 janvier 1934, d'un fragment d'amphore portant une inscription peinte en noir ${ }^{3}$. La description de la pièce, rédigée après l'achèvement des travaux de restauration et insérée à la fin du mois de juin 1934, détaille la décoration pariétale.

Malgré leur mauvais état de conservation, les vestiges d'enduits en place fournissent tous les éléments nécessaires à la restitution de la structure décorative. En zone inférieure, l'enduit presque intégralement conservé permet d'observer que sa structure est identique sur toutes les parois : un panneau central rouge bordeaux encadré de deux massifs piédestaux ocre jaune. En revanche, seule une lecture attentive permet de repérer les motifs picturaux, très usés et concrétionnés : ainsi le panneau rouge central est couronné par un plafond à caissons reposant à l'arrière-plan sur deux supports, et son centre est occupé par un pseudo-édicule schématique enfermant, sur le mur sud, un haut vase cylindrique d'où jaillit une sorte de tige sinueuse ${ }^{4}$, et sur les autres murs un motif peu discernable. Sur les murs nord et sud, une fine guirlande relie cet édicule aux supports du plafond à caissons ; sur les murs est et ouest c'est une bordure ajourée horizontale. Le traitement des massifs piédestaux peut être restitué grâce aux sections les plus lisibles: leurs moulures inférieures se traduisent par une série d'horizontales rouge-bordeaux plus ou moins larges, surmontées par une rangée de feuilles d'eau ornant environ un tiers de la hauteur du corps des piédestaux. La limite supérieure de ces massifs architecturaux, apparemment dépourvue de moulures, ne comporte pas non plus de corniche, mais la présence de celle-ci est suggérée

2 Giornale degli scavi d'Ercolano (GSE), 1932-1934, archives du Musée archéologique de Naples.

3 CIL, IV, 10825, attribuée par erreur à la Casa sannitica V, 1-2.

4 Ce motif pourrait rappeler le vase dionysiaque MANN inv. n 9965 d'où sort un serpent. 
par un large tracé rouge-bordeaux sinueux et irrégulier censé représenter l'ombre portée par le couronnement.

En zone médiane, la structure tripartite reprend celle de la zone inférieure, sans toutefois qu'elle suive parfaitement l'alignement des axes verticaux. Un champ central ocre jaune est encadré de deux échappées architecturales à fond blanc qui prennent appui sur les piédestaux de la zone basse. Pour restituer l'aspect du champ central, on ne dispose que d'une donnée : les traces d'entablement en saillie sur les murs est et nord indiquent la présence d'un cadre architectural sous la forme d'un édicule, dont aucun élément ne subsiste pour en préciser l'aspect. La restitution proposée reste donc parfaitement neutre. Une autre incertitude touche la présence de tableaux ou de figures au centre des murs nord, sud et ouest, très lacunaires. Sur le mur oriental, en revanche, l'espace disponible sous la fenêtre a permis d'insérer une vignette à peu près disparue aujourd'hui (pl. VII.1).

Les échappées de la zone médiane ne sont conservées que sur le mur est ; mais les lambeaux de leur couronnement subsistant sur le mur sud témoignent de la répétition identique du motif sur tous les murs. Elles comportent deux niveaux et affectent la forme d'un haut édicule à épi, couronné d'un fronton vert en accolade ; l'édicule est fermé par une barrière à croisillons derrière laquelle se tient une figure féminine debout sous un plafond à caissons, et il débouche sur un espace ouvert où apparaît un portique fuyant. Le second niveau, peu développé, s'ouvre, au-delà d'une baie cintrée, sur une section de portique frontale. L'entablement qui surmonte l'ensemble de l'échappée est traité en alternance de bucranes très stylisés et de rosettes, identique, mais à une échelle légèrement supérieure, à celui qui surmonte l'édicule du premier niveau. L'épi qui flanque chacune des échappées en limite avec le champ central est constitué de deux colonnes ioniques cannelées sans base visible et portant un entablement fuyant.

La zone supérieure à fond blanc, également tripartite, s'organise de part et d'autre d'un édicule frontal à entablement plat, couvert d'un plafond à caissons et dont le fond semble bleu-gris : comme la seule attestation en est un lambeau sur le mur sud, on ne peut affirmer s'il renfermait une figure ou un motif. Les zones latérales se subdivisent à leur tour; le tiers central est constitué d'un pseudoédicule (vert sur les murs sud et nord, bleu sur les murs est et ouest) à fronton linéaire en accolade abritant un griffon en vol ; il se prolonge en un second niveau délimité par de fins candélabres végétaux et abritant un Amour en vol. Griffon et Amour sont aujourd'hui à peine discernables. Dans les tiers latéraux, une bordure horizontale très effacée sert de support à un oiseau (vers les angles) et un paon (vers l'édicule), sous une guirlande en arc.

La restitution des édicules et pseudo-édicules de la zone supérieure se fonde d'une part sur la reprise symétrique des éléments conservés, et d'autre part sur la correspondance dimensionnelle avec les subdivisions de la zone basse. L'apport des rares archives et des fragments prélevés au XVIII siècle enrichit quelque peu ce cadre général. Prises dans les années 1970, quelques photographies permettent 
de préciser les détails de modénature, la silhouette des figures féminines dans les architectures, l'aspect des échappées architecturales. Sur le mur nord, la vignette, encore lisible il y a une quarantaine d'années, représente une figure féminine demi-couchée, tournée vers la droite, son coude droit appuyé sur un rocher, dans l'attitude d'une divinité fluviale ou d'une source. Elle revêt un intérêt d'autant plus grand que, les personnages des échappées mis à part, il s'agit de la seule figuration signifiante dans cette pièce. Insérée dans le mur mitoyen de celui du nymphée, elle renvoie alors, de toute évidence, à la connotation de celui-ci et au ruissellement de la fontaine voisine.

Deux fragments prélevés au XVIII ${ }^{\mathrm{e}}$ siècle ont pu être repérés grâce à leurs pendants in situ : ce sont le paon et l'oiseau trouvés le 26 mars 1746 puis réunis sous un même numéro d'inventaire (MANN inv. ${ }^{\circ} 8703$ ). Ils sont restitués en zone supérieure à droite du mur sud. Outre leur meilleure lisibilité, ils permettent de compléterle décor : labordure ajourée surlaquelleils posent, complètement effacée in situ, a gardé intact son motif de postes qui a donc été rétabli graphiquement sur l'ensemble de la zone supérieure.

Ainsi restitué, le décor de cette pièce s'inscrit dans une parfaite cohérence avec ceux de l'atrium et du tablinum ; la zone basse se structure de la même façon en grands panneaux rouge bordeaux alternant avec des massifs architecturaux qui supportent les échappées de la zone médiane, ces dernières traitées de façon scénographique. Par ailleurs, on observe de grandes similitudes de mise en œuvre entre ces pièces qui ont de toute évidence fait l'objet d'une même campagne décorative et trahissent l'intervention d'un même atelier (pl. VIII.1).

D'autre part, on constate que, de façon générale, ce décor qui vise à l'effet par l'aspect imposant de certains de ses éléments architecturaux, a pourtant été exécuté de façon assez peu soignée ; de forts écarts dimensionnels (allant jusqu'à $10 \mathrm{~cm}$ dans une pièce de dimensions aussi réduites) se remarquent entre les zones symétriques d'un même mur; les candélabres de la zone supérieure sont mal positionnés par rapport au sommet des pseudo-édicules censés les supporter ; le rendu des faces fuyantes des gros piédestaux est déficient (même compte tenu des lacunes), et l'absence de corniche supérieure, simplement remplacée par son ombre portée, laisse à désirer. D’un point de vue technique, la peinture a été posée sur un enduit trop sec, ce qui a amené la perte complète des détails : les bordures ajourées, les entablements, les filets verticaux de la zone médiane du mur est, les motifs du socle, ont disparu pratiquement sans laisser de traces. En zone supérieure du mur sud, le pseudo-édicule vert clair qui abrite le griffon laisse transparaître des traits de pinceau préparatoires assez désordonnés.

L'impression générale que l'on retire de ce décor nuance donc l'opinion d'A. Maiuri, qui voyait dans cette maison l'une des réalisations les plus fastueuses ${ }^{5}$.

5 A. Maiuri, Ercolano, I nuovi scavi (1927-1958), Rome, 1958, p 397. 


\section{Le décor de l'atrium}

La fouille de l'atrium 10 (8,87 x 7,20 m), alors numéroté 3, est mentionnée entre le $1^{\mathrm{er}}$ mars et le 17 juin 1933. Tout au long de ce dégagement, les descriptions sont limitées à l'essentiel, lorsque des objets, inventoriés ou non, sont découverts. Une attention plus soutenue est portée à l'autel du culte domestique situé dans l'angle nord-ouest de la pièce et sur le parcours d'un cunicolo du XVIII' siècle. Des fragments de pinakes de marbre ${ }^{6}$ suscitent l'enthousiasme d'A. Maiuri qui évoque l'idée d'une décoration pariétale inachevée ; l'inscription purpura (CIL IV, 10 480), peinte au-dessus d'un tableau mythologique en zone supérieure de la paroi occidentale, laisserait supposer que la décoration était en voie d'achèvement, seule la bordure des cadres devant encore être mise en couleur.

Le décor de l'atrium est décrit, par A. Maiuri, comme

“ la più fastosa e scenografica decorazione d'atrio (...) dovuta ad un artista di grande ricchezza coloristica, di sicura bravura, di ardimento e di fantasia inventiva, di un artista, infine specializzatosi, potremmo anche dire, nel genere della pittura architetturale barocca, e non alieno, come accade invece nei correnti decoratori della casa ercolanese, dall'inserire soggetti figurati e figure isolate e motivi animalistici nel campo della decorazione architettonica ${ }^{7}$. »

L'enthousiasme de cette description ne peut que faire regretter non seulement sa quasi-disparition, mais encore l'indigence de la documentation graphique et photographique. Dans son état actuel, il ne reste que des lambeaux de décor, la plupart situés en zone basse, quelques-uns en zone médiane sur les murs nord et sud. De la zone supérieure, il ne subsiste qu'un fragment sur le tiers droit du mur est. Sur le cliché des années 1930 publié par A. Maiuri, une grande plaque d'enduit adhérait encore sur le mur est, au-dessus de la porte de la pièce $4^{8}$; elle a aujourd'hui disparu sans qu'un relevé ou une photographie de détail en ait été pris. Sur le même mur, une autre plaque surmontait les portes donnant sur l'andron et sur le triclinium 7 : seul un lambeau est encore partiellement lisible. La même photographie montre, à l'extrémité gauche du mur sud, un auvent qui protège une grande plaque en zone haute, disparue elle aussi.

6 GSE 1933 : « 20 aprile. [...] ». A. MaIURI, Ercolano, 1958, p. 394-396; MANN inv. n 150210 et 15211 : I. Bragantini et V. Sampaolo, La pittura pompeiana, Naples, 2009, p. 119, $\mathrm{n}^{\circ}$ 14a. Sur ces pinakes :A. DARdenay et al., “ Des archives aux restitutions architecturales et décoratives ", Chronique des activités archéologiques de l'École française de Rome, 2016, 18-20. [En ligne], Les cités vésuviennes, mis en ligne le 09 juin 2016, consulté le 09 juin 2016. URL : http://cefr.revues.org/1588

7 A. Maiuri, Ercolano, p. 397.

8 A. Maiuri, Ercolano, fig. 332, p. 396. 
La méthode que nous avons suivie a donc consisté ici tout d'abord à tirer parti des éléments encore visibles en zone basse pour fixer le rythme des panneaux et inter-panneaux, puis à exploiter les faibles traces lisibles en zone médiane, enfin à s'appuyer sur les archives photographiques pour restituer l'essentiel de la zone supérieure.

Le mur nord (L : 8,87 m), le seul à ne pas être interrompu par des ouvertures, ne présente pratiquement pas de solution de continuité dans les enduits de sa zone basse. Malgré l'érosion de la couche picturale, il a donc été possible de relever les mesures des panneaux et des compartiments, et de les restituer sur l'orthophotographie. La partition décorative fait alterner de grands panneaux rouge bordeaux et des panneaux ocre jaune plus étroits, eux-mêmes animés par un compartiment rouge bordeaux : ils sont à comprendre comme de gros piédestaux dans lesquels s'inscrit une table de couleur contrastée, à couronnement cintré aux extrémités est et ouest de la paroi, et en bâtière pour les deux éléments centraux ; ces compartiments s'ornaient de motifs dont seul un vase en forme de cratère est conservé à l'extrémité droite du mur nord. Entre ces piédestaux, les panneaux rouges sont recoupés par des filets horizontaux et verticaux formant un encadrement au centre duquel une ligne de sol porte un animal ou un monstre : l'un d'eux, apparemment un capriné tourné vers la gauche, est encore partiellement visible sur le panneau central, tandis que sur le panneau gauche, un quadrupède ailé à queue recourbée est tourné vers la droite. À juste titre, A. Maiuri voyait ce soubassement, sans doute plus lisible à l'époque, comme l'évocation du proscenium d'un pulpitum de théâtre.

La restitution de la zone médiane s'appuie d'une part sur ce rythme, d'autre part sur les vestiges faiblement lisibles à l'extrémité gauche du mur nord, enfin sur la plaque repositionnée sur le mur sud. S'y ajoute la notation d'A. Maiuri selon lequel les champs médians étaient constitués de grands édicules s'ouvrant entre de fastueuses perspectives architecturales. On peut donc établir que les piédestaux ocre jaune portaient des architectures sur fond blanc dont on lit encore la succession de colonnes ou de supports ocre jaune, rose, violacé, gris, sans que l'on puisse en dire davantage sur leur organisation spatiale. Ces échappées s'ouvrent dans des panneaux aujourd'hui décolorés, noirs à l'origine, alignés sur la largeur des panneaux rouge bordeaux du soubassement ; un large encadrement ocre jaune formait transition avec les échappées.

Rien ne subsiste sur le mur nord de l'aspect supérieur de cette zone et du couronnement des panneaux. Cependant, une plaque repositionnée au centre du mur sud fournit quelques données : malgré son effacement, on y lit encore les traces d'un plafond à solives en perspective ménageant un opaion central à fond clair, sans doute bleu, sous un couronnement cintré. Immédiatement audessous, dans le champ noir, l'angle supérieur droit d'un tableau est conservé : par symétrie, on peut en restituer la largeur $(0,54 \mathrm{~m})$. 
Toutefois, le repositionnement de cette plaque lors des fouilles des années $1930^{\circ}$ est légèrement fautif, et pour respecter la correspondance avec la partition du soubassement, elle doit être décalée de 0,20 m vers la gauche. De plus, on note que la plaque se présente en deux fragments non jointifs à l'origine, de sorte qu'il faut abaisser celui qui conserve l'angle du tableau afin de replacer celui-ci, sinon au centre, du moins à l'intérieur du champ de l'édicule conformément aux habitudes décoratives romaines.

Cet édicule se trouvant au centre du mur sud, il est certain que son pendant sur le mur nord revêtait le même aspect. Quant aux édicules latéraux, ils devaient avoir un traitement voisin, sinon tout-à-fait identique ; en l'absence de données, il leur a été restitué un simple entablement plat. Ils étaient au nombre de deux sur le mur nord, probablement un seul sur le tiers gauche du mur sud (interrompu par trois portes), un sur le tiers gauche du mur ouest et un, sans doute un peu plus étroit, sur le tiers droit, au-dessus du laraire ; à l'est, le mur interrompu par quatre portes ne laissait aucun espace pour un panneau architectural, et la perte du soubassement nous prive d'indications (pl. VIII.2).

Sur la foi des archives de fouilles ${ }^{10}$ qui mentionnent un tableau mythologique sur le mur ouest et des vestiges de figures sur les murs sud et est, on peut admettre que des tableaux ornaient les édicules latéraux. Comme il n'en reste pas trace et que l'enquête dans les journaux de fouilles des Bourbons n'a permis de repérer aucun prélèvement ${ }^{11}$, ces tableaux sont symbolisés dans la restitution graphique par de simples contours.

La zone supérieure (H. totale : 3m) est aujourd'hui complétement perdue. Cependant un cliché en noir et blanc de 1935 (Alinari 43124) documente le mur est et apporte des éléments sur sa structure (fig. 1). En premier lieu, il permet de compléter l'aspect de l'entablement qui couronne la zone médiane: assez complexe, il s'articulait en une alternance de compartiments couverts d'un plafond

9 Voir supra, p. 1 ; les équipes mises en place par A. Maiuri reconstruisaient au fur et à mesure des fouilles les parois fragilisées, ce qui les amenait à prélever les enduits pour les repositionner sur les murs ainsi consolidés. Sur la méthode de fouille : A. MaIURI, Ercolano, 1958, p. 21.

10 GSE du $1^{\text {er }}$ mars 1933 repris dans la Descrizione generale, ambiente 3 : "Sulla parete ovest, che conserva maggiore quantità di stucco, sul campo alto si nota una prospettiva con due centauri e nel centro un quadro con figure mitologiche. Anche sulla parete sud ed est vi sono avanzi di figure ». Au-dessus du tableau mythologique se trouvait l'inscription PVRPVRA.

1 A. Allroggen-Bedel, "Ercolano. Dal sito agli archivi, dal museo al sito », in A. Coralini, Vesuviana: Archeologie a confronto. Atti del convegno internazionale (Bologna, 14-16 gennaio 2008), Bologne, 2009, p. 167-179 ; ead., " I documenti settecenteschi come strumenti per lo studio degli scavi ercolanesi », Anabases 26 (2017), p. 103-116. 


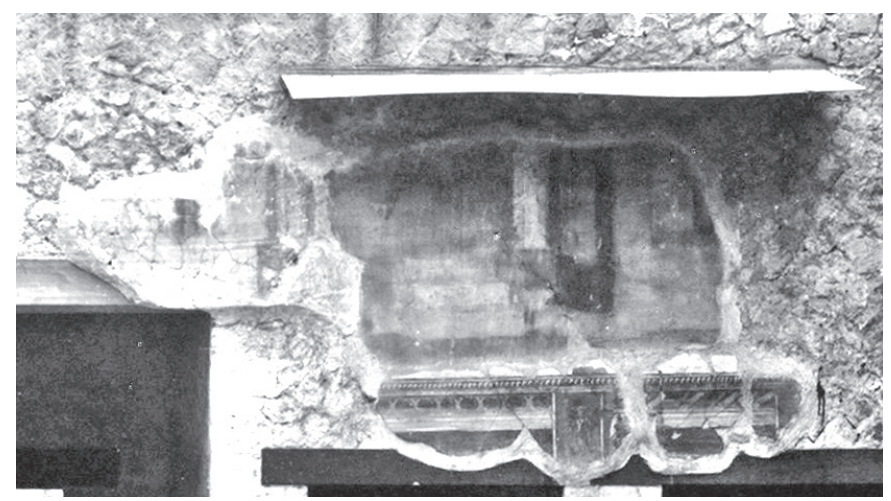

Fig. 1. Maison de Neptune et Amphitrite, atrium 10, mur est zone supérieure. Photo Alinari 43124 (détail).

à caissons (au-dessus des échappées) et de corniches à consoles (au-dessus des champs noirs). De petits dés scandaient cette alternance et portaient une figure hybride ailée au corps terminé en feuilles. Il n'en subsiste qu'une au-dessus de la porte du couloir 9, de sorte que, en l'absence d'autre donnée, il a été choisi de la restituer partout à l'identique, en n’opérant que des retournements symétriques.

$\mathrm{Au}$-dessus de cet entablement, la photo d'archives révèle, à droite du mur est, une série de membres architecturaux dont les valeurs de gris ne facilitent pas l'interprétation ; retravaillé numériquement, décrypté en fonction du répertoire architectural des décorateurs campaniens, ce cliché a cependant permis de restituer l'aspect global de la zone supérieure. L'élément le plus manifeste est un escalier suivi, à droite, d'un étroit pan frontal puis d'une paire de colonnes en plan fuyant réunies par un muret. À gauche de l'escalier, on discerne l'amorce d'un pan de paroi frontale. Enfin, au sommet de l'escalier, apparaît la forme d'un pied sous un vêtement incurvé, ainsi que la faible trace d'un second. Ces quelques éléments signalent clairement un dispositif “ scénographique ». La figure, vraisemblablement masculine en raison du pan de toge, devait avoir une hauteur d'environ $1 \mathrm{~m}$ et l'architecture qui l'encadre environ 2,20 m. En l'absence de couronnement, il est malaisé de caractériser ce bâtiment, qui appartient au type du pavillon, de même que celui qui le suit à l'angle du mur.

La photo d'archives témoigne à l'extrémité gauche du mur est de vestiges architecturaux symétriques aux précédents, avec des dimensions un peu distendues : le pavillon d'extrémité est large de $0,79 \mathrm{~m}$, contre les $0,65 \mathrm{~m}$ de celui de droite. Il est séparé du pavillon à escalier par une zone où se lisent trois montants verticaux reliés par un compartiment à bord supérieur plus ou moins concave : il pourrait s'agir de la base d'un trépied dont on ne connait ni l'aspect ni la hauteur.

La synthèse de ces données laisse, au centre du mur est, un espace de 1,20 m dont le décor est tout à fait inconnu, de même que nous ignorons tout du sommet 
de la zone supérieure sur une hauteur de 0,75 m. Ces surfaces ont donc été laissées vides dans la restitution.

Si tout le parti possible a pu être tiré du cliché en noir et blanc, celui-ci laisse entière la question des couleurs des éléments architecturaux. Aussi le choix a-t-il consisté à traiter ces éléments au trait sur un fond rouge uni, dans la mesure où la description des archives de fouilles insiste sur cette couleur dominante dans l'atrium (pl. VIII.2).

Les données manquent pour restituer les murs nord et sud, plus longs; le schéma des murs est et ouest a donc été reproduit en élargissant le champ central vide (3 m) au-dessus de l'édicule central médian.

La structure décorative de l'atrium confirme le jugement d'A. Maiuri : l'important dispositif architectural constitué par de larges échappées à fond clair encadrant les panneaux bidimensionnels est moins courant dans les atria que dans d'autres pièces publiques ou de réception (tablinum, oecus, triclinium) ; en effet, la hauteur considérable des parois et leur surface discontinue irrégulièrement interrompue par des portes, se prêtent mal aux effets « scénographiques ». Citons, cependant le vaste atrium 4 de la maison VIII 2, 18-21 à Pompéi, aujourd'hui perdu mais documenté par des photographies et par une planche de Niccolini ${ }^{12}$ : comme dans celui de la Casa di Nettuno ed Anfitrite, l'un des murs (nord) disposait d'une surface continue. Le choix de ce système décoratif ambitieux signale donc, de la part du commanditaire, une volonté d'effet.

Cette volonté se manifeste également par l'abondance de panneaux noirs. Or on sait que ce choix chromatique implique une mise en œuvre complexe : le noir de carbone étant incompatible avec la technique de la fresque, il faut colorer l'enduit, puis poser une première couche, généralement rouge, enfin étendre le pigment noir mélangé à de la colle animale ${ }^{13}$.

C'est sur le site d'Herculanum que les commanditaires ont le plus apprécié les murs noirs : les Case dei Cervi ou del Salone nero en sont les exemples les plus frappants, et ceci s'inscrit dans la cohérence de leur goût pour les matériaux rares et précieux (opus sectile) et les fonds colorés coûteux ou de réalisation complexe ; rappelons les murs bleus de la Casa del Gran Portale ou celle dell'Atrio a mosaico, et les murs verts de la Casa Sannitica.

La restitution des décors de la Casa di Nettuno ed Anfitrite affronte les défis habituels que sont l'accélération de la dégradation, les difficultés d'identification et de localisation des prélèvements au sein des collections du MANN, les lacunes de la documentation. Toutefois, les caractères mêmes de ce décor facilitent

12 F. et F. Niccolini, Le case ed i monuenti di Pompei disegnati e descritti, Naples, 1896.

13 Vitr. 7, 10, 1-2 ; Pline Nat 35, 25 ; D. Burlot et H. Eristov, « Le fond noir en peinture, marqueur du luxe et gageure technique », Pictor 6 (2017), 225-249. 
quelque peu la tâche. En effet, l'ensemble, très cohérent, est de toute évidence l'œuvre d'un même atelier qui applique les mêmes recettes et utilise le même répertoire dans toutes les pièces : en particulier le dispositif scénographique du pavillon à escalier ou à barrière abritant une figure se retrouve dans le triclinium 7, le cubiculum 4, l'atrium et le tablinum. De plus, les choix du commanditaire l'ont porté, on l'a vu, à des solutions riches d'effet dans une certaine économie de moyens ; en particulier un système décoratif identique orne les quatre parois d'une même pièce, ce qui a pu être vérifié dans le cubiculum 4, contrairement aux habitudes romaines consistant à différencier, plus ou moins légèrement, les parois perpendiculaires. De ce fait, la reprise, sur tous les murs de l'atrium, du système observé sur une des parois, trouve sa légitimité.

Outre les deux pièces commentées ici, les fauces, la cella atriensis 2, le triclinium 7, le couloir 9, la pièce 6 et celle (22) qui la surmonte à l'étage, ainsi que la façade ont fait l'objet de restitutions qui confirment l'homogénéité du projet décoratif, au point que des motifs identiques ont pu être repérés dans le triclinium et les mosaïques du nymphée. En revanche, des vestiges d'une phase antérieure subsistent dans le décor peint du nymphée et surtout dans la boutique, décor occulté partiellement par l'installation de la mezzanine et la construction des pièces d'étage.

La démarche de restitution adoptée ici s'appuie donc tant sur les données visibles (même difficilement) in situ, que sur les archives de fouilles, et sur les panneaux prélevés et conservés au MANN. Le défi, certes ambitieux, de redonner une visibilité aux décors de l'ensemble d'une maison contribue à mieux caractériser les spécificités d'Herculanum par rapport à sa voisine Pompéi, à appréhender les choix et les références du commanditaire, ainsi que le fonctionnement des ateliers actifs sur ce chantier. Si, dans sa volonté de reconstruire une cité antique à ciel ouvert, Maiuri a innové techniquement et mis en œuvre des moyens considérables, ses méthodes n'ont pas été sans défauts, et les restitutions des décors pariétaux tentent, dans une certaine mesure, d’y remédier.

\author{
Hélène Eristov \\ CNRS-ENS-UMR 8546 \\ 45 rue d'Ulm, 75005 Paris \\ helene.eristov@ens.fr
}

\title{
Marie-Laure Maraval
}

DAR, Université Toulouse 2 Jean-Jaurès 5 allées A. Machado

31058 Toulouse Cedex 9 mmaraval@univ-tlse2.fr 
Anabases 27 (2018), pl. I-XII.

\section{Restituer Herculanum II planches}

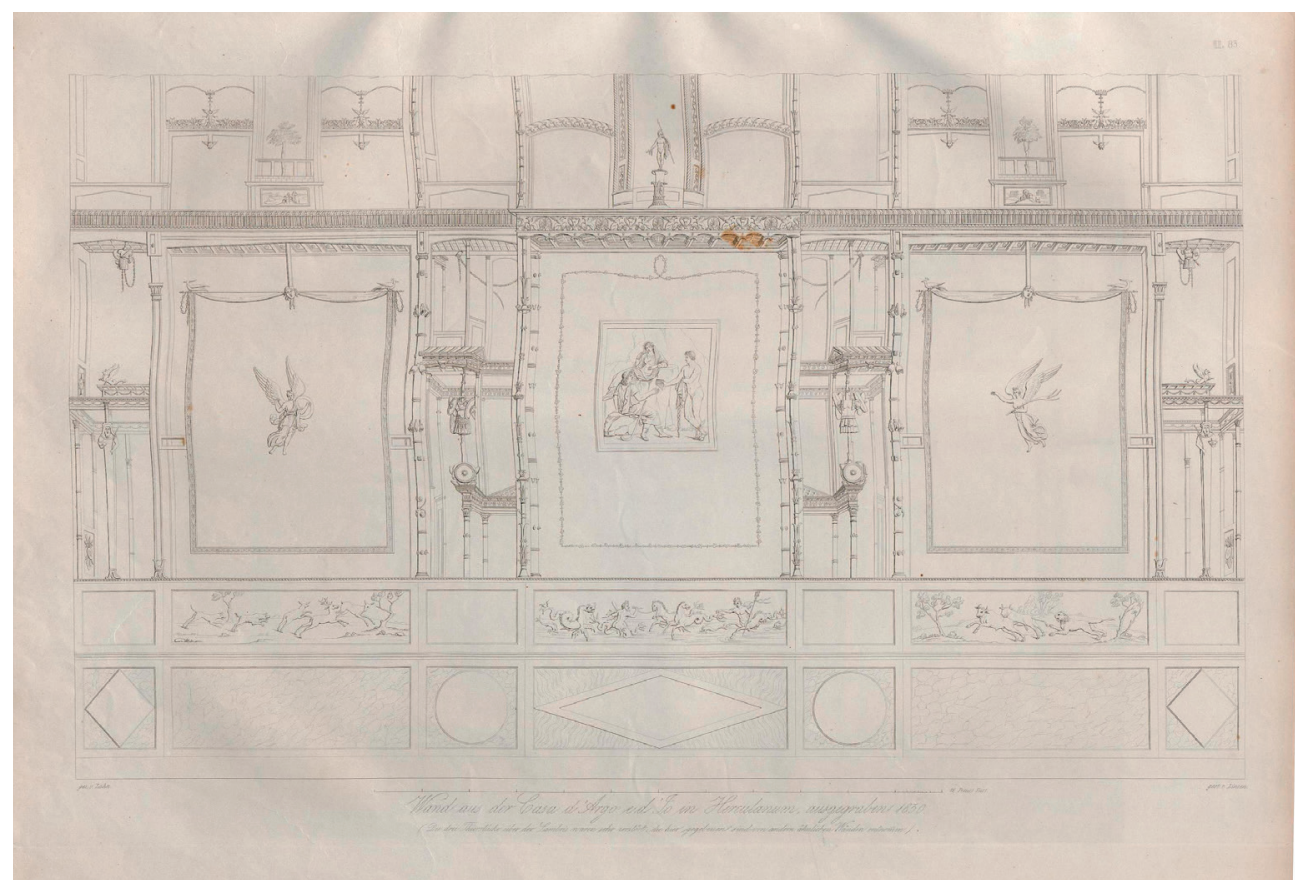

1. Paroi du triclinium, d'après W. Zahn, Les plus beaux ornements, tableau 83. 


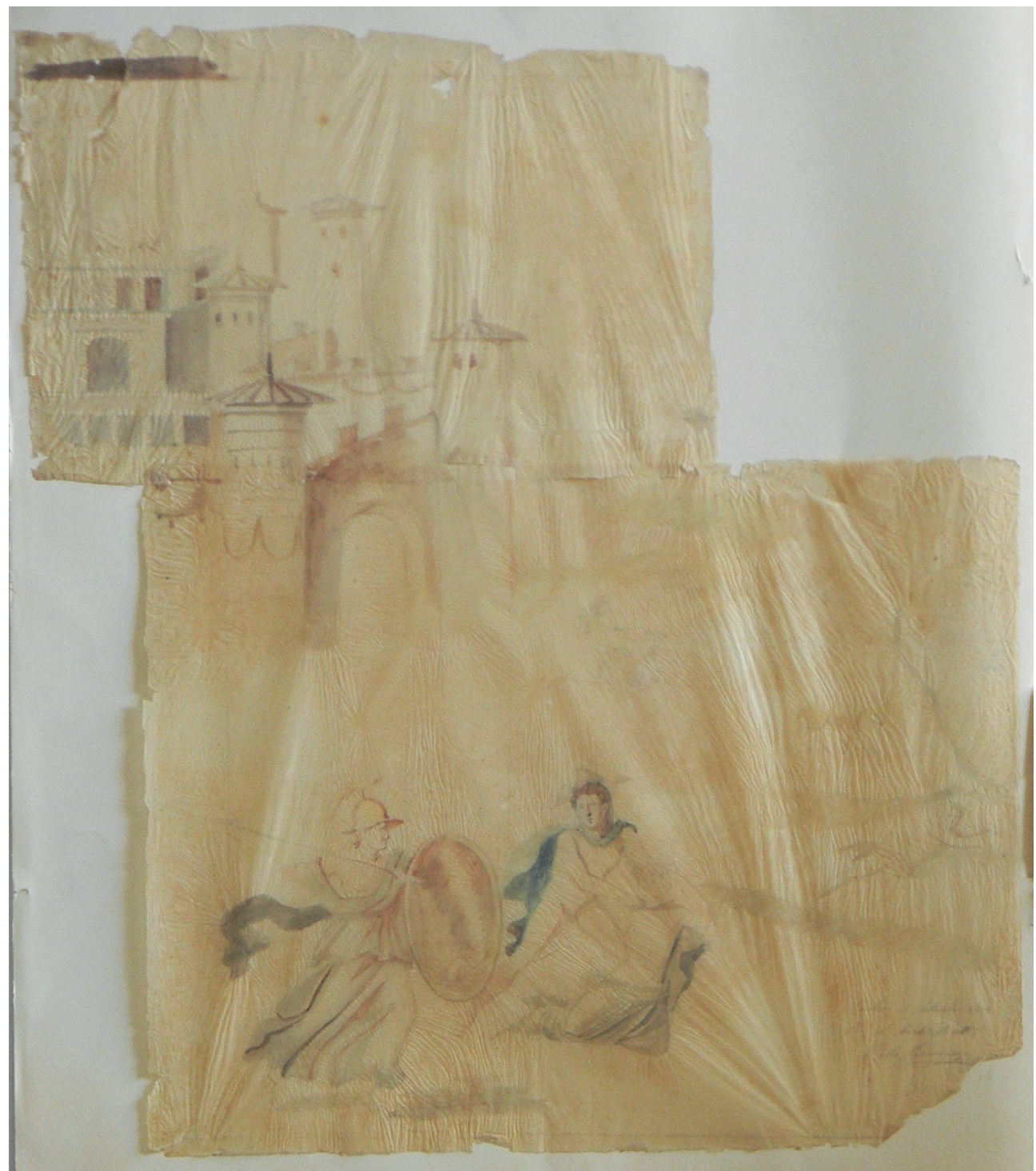

1. Aquarelle réalisée en 1828. Cartella Ercolano RA/10 n. 5530, Archivio disegni della Soprintendenza per $i$ Beni archeologici di Napoli. Avec l'aimable autorisation de la Soprintendenza et du Ministero dei Beni e delle Attività Culturali e del Turismo. 


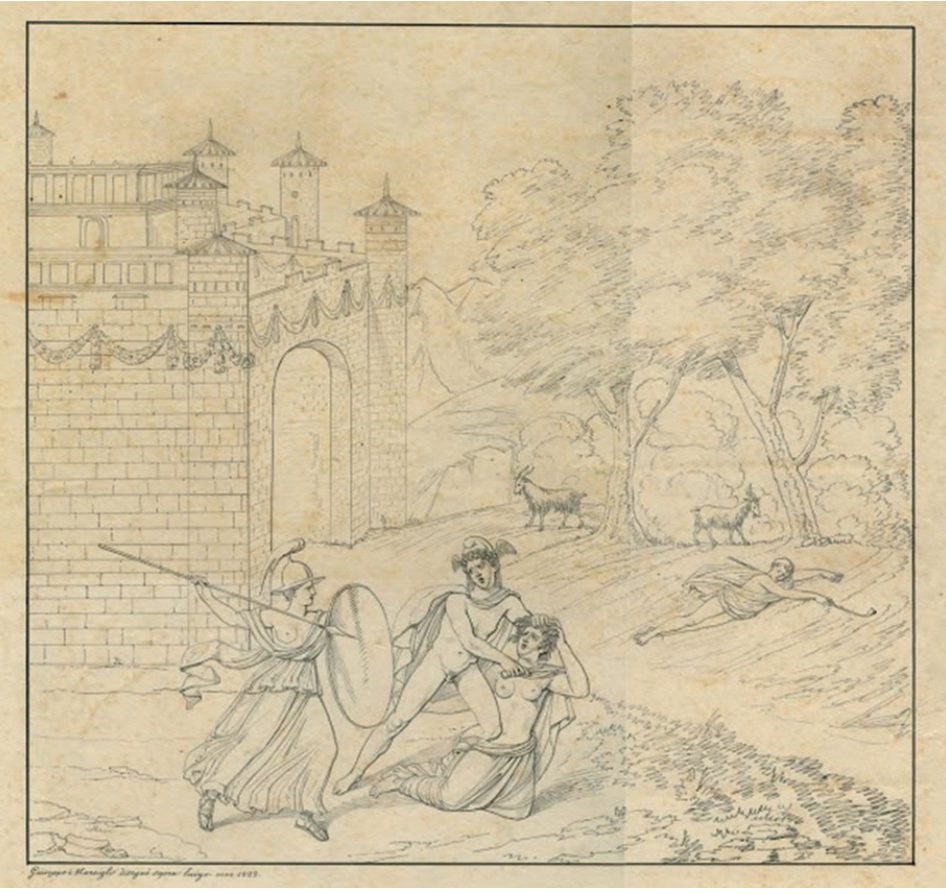

1. Dessin

au crayon exécuté

en 1829.

Cartella Ercolano

RA/10 n. 5530,

Archivio disegni della

Soprintendenza per

i Beni archeologici

di Napoli.

Avec l'aimable

autorisation de

la Soprintendenza

et du Ministero dei

Beni e delle Attività

Culturali e del

Turismo.

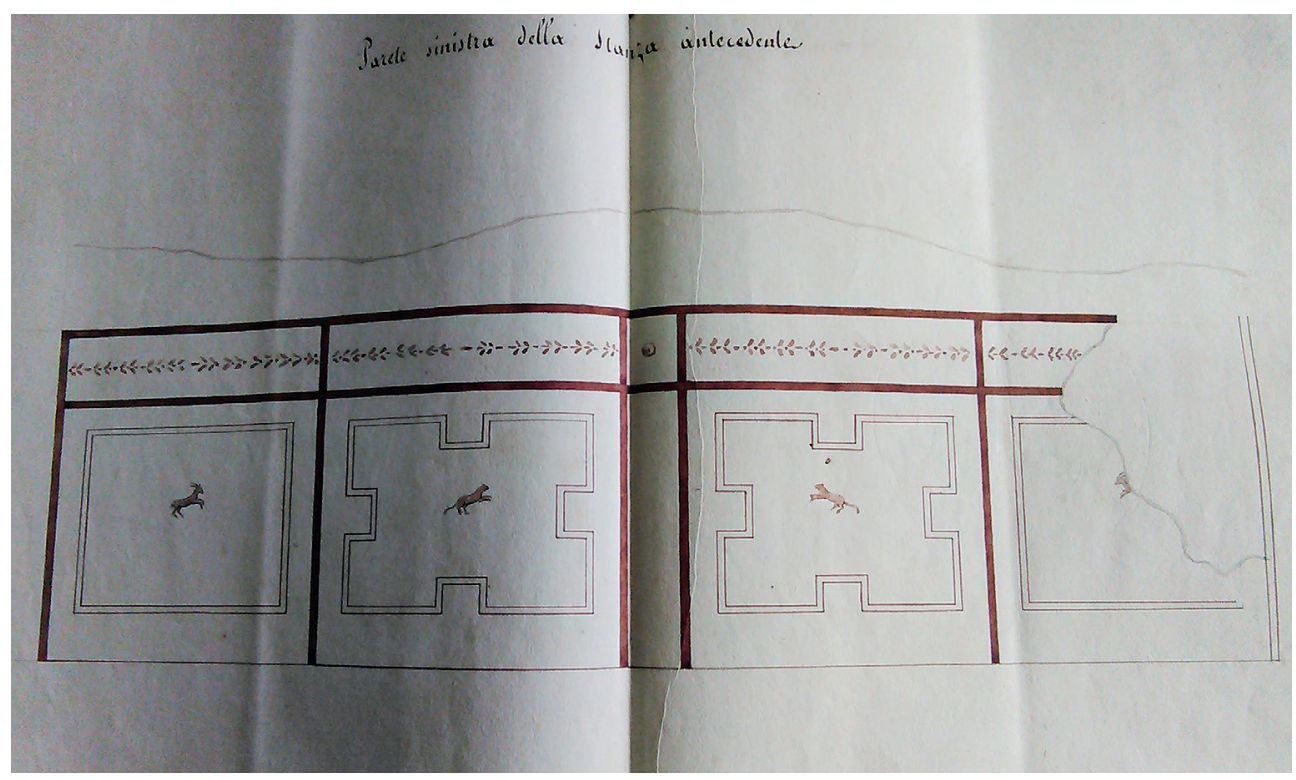

2. Dessin du décor d'une paroi de l'étage supérieur. Archivio storico della Soprintendenza per i Beni archeologici di Napoli ; fasc. VII C9, 3. Avec l'aimable autorisation de la Soprintendenza et du Ministero dei Beni e delle Attività Culturali e del Turismo. 


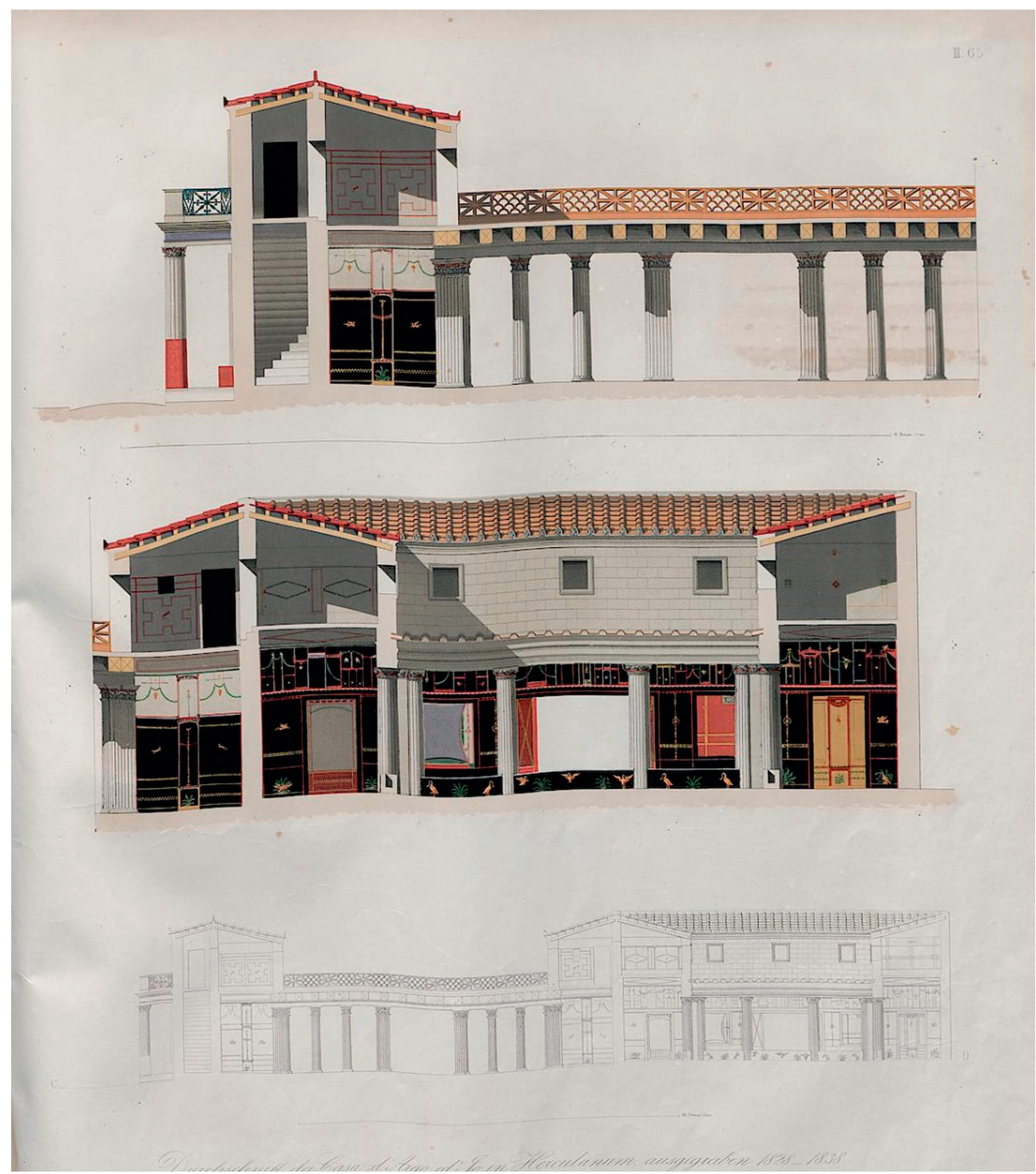

1. Coupe du mur de l'étage supérieur et du rez-de-chaussée. W. Zahn, Les plus beaux ornements, tableau 65 
1. Statuette de Dionysos provenant de « l'atelier du plumbarius »(VI, 12) à Herculanum. D’après T. Budetta - M. PAgano, Legni e bronzi, fig. p. 110.
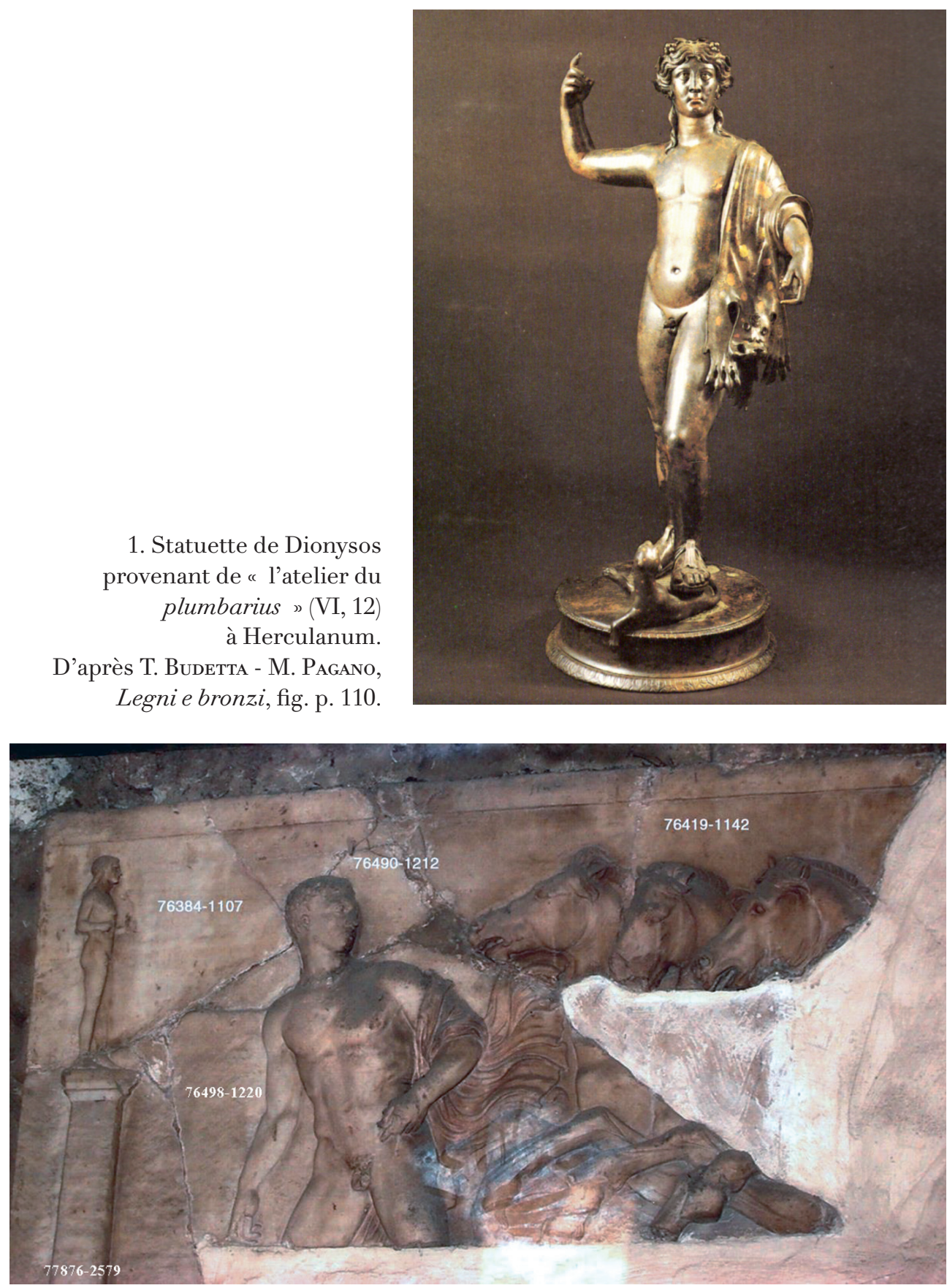

2. Herculanum, Casa del Bel Cortile : les fragments du “ relief A » et leurs numéros d'inventaire. Cliché N. Monteix. 
1. Herculanum, Casa del Bel Cortile : le fragment 1220. Cliché N. Monteix.
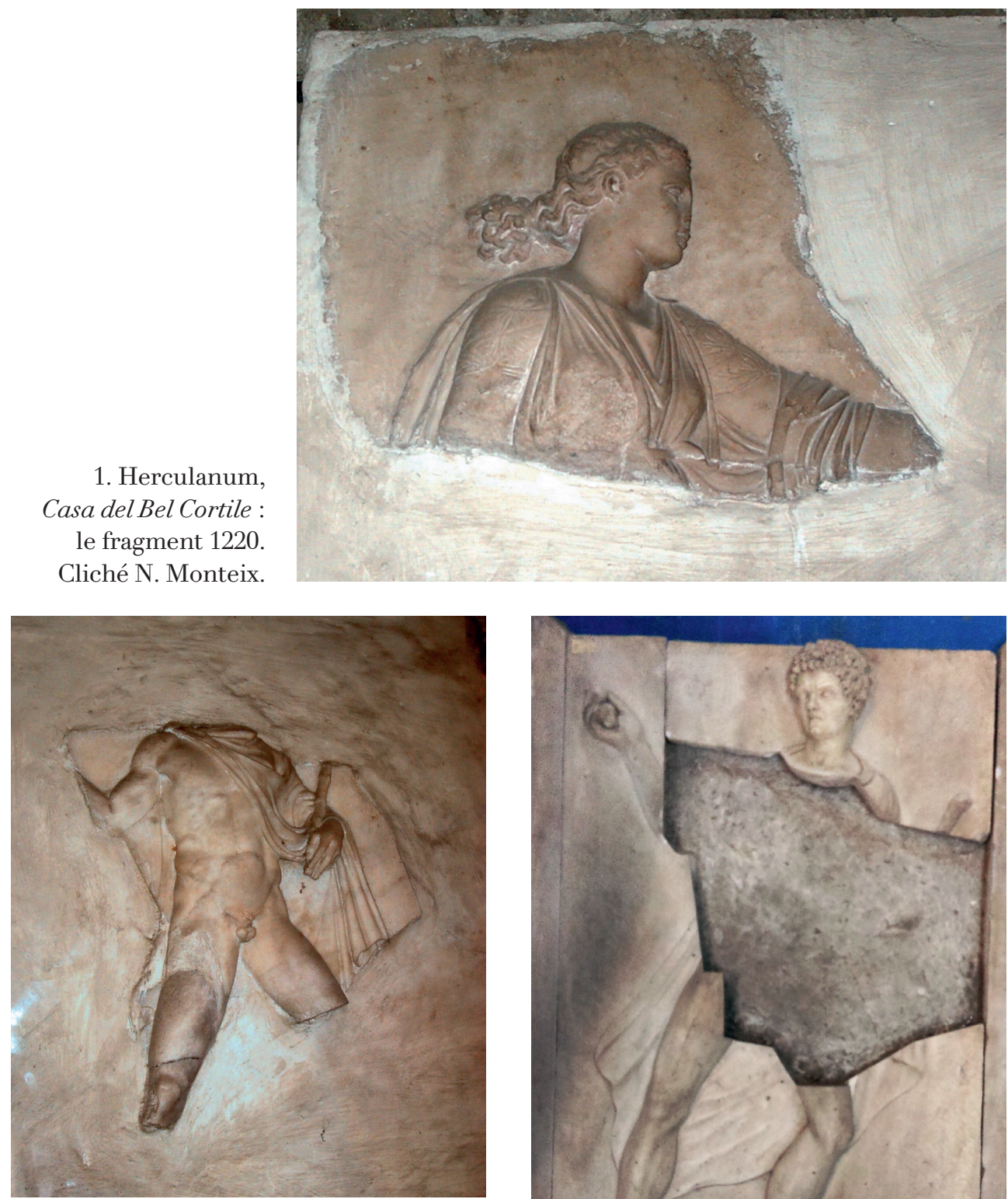

2. Herculanum, Casa del Bel Cortile, “ relief B » : le fragment 1215.

Cliché N. Monteix.

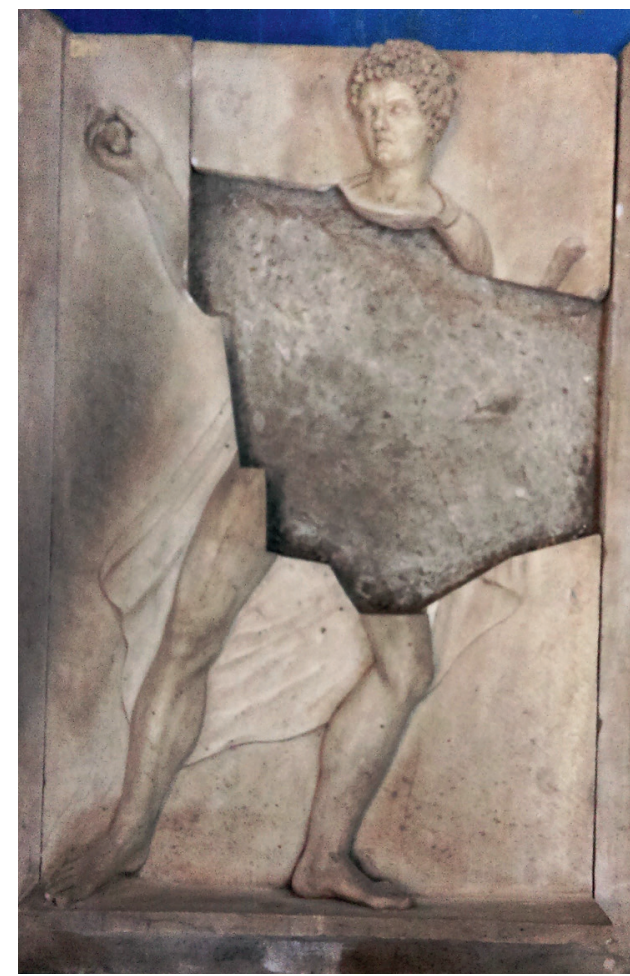

3. Naples, MANN, inv. 6680, relief dérestauré, après prélèvement du fragment appartenant au " relief A »d'Herculanum. Cliché E. Rosso. 

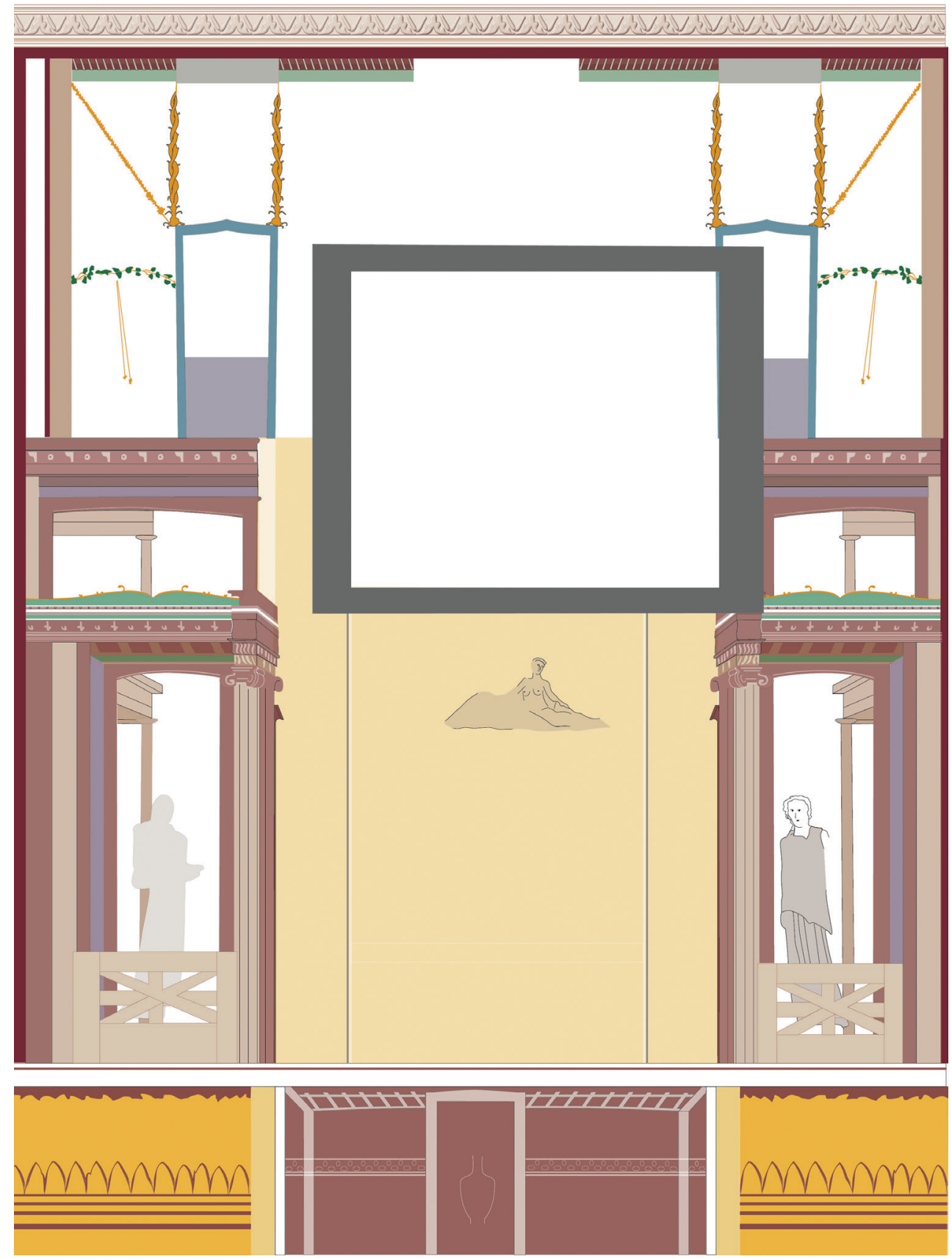

1. Maison de Neptune et Amphitrite, pièce 4, mur est, restitution (infographie M.-L. Maraval). 


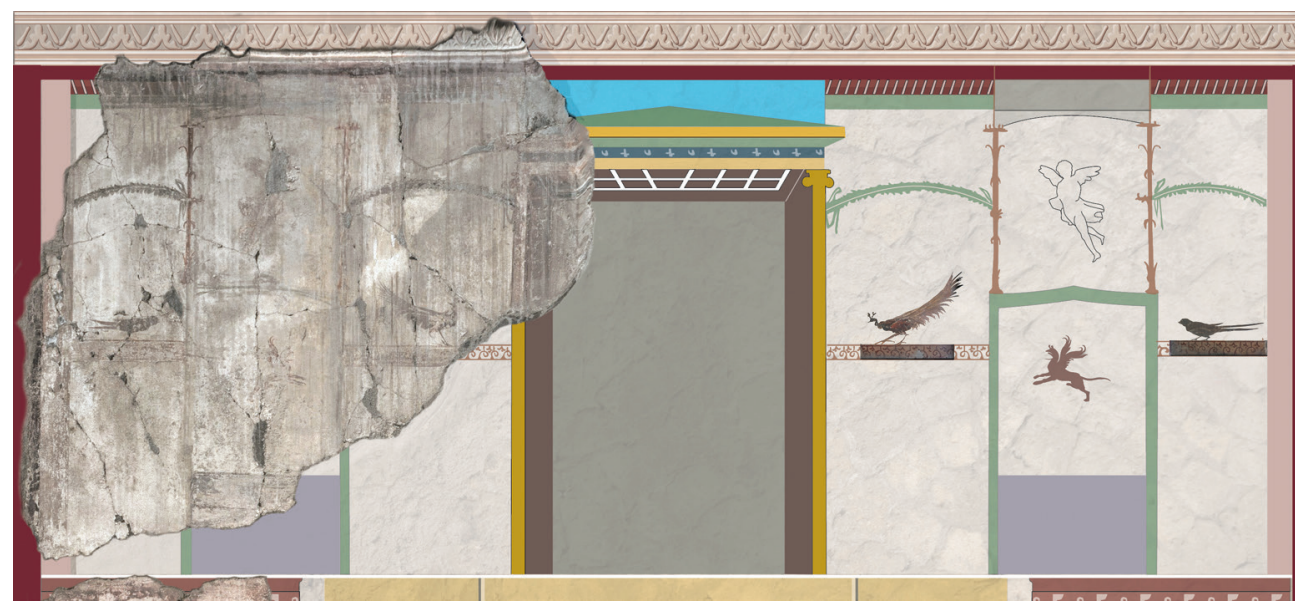

1. Maison de Neptune et Amphitrite, pièce 4, mur sud, zone supérieure, restitution avec les enduits in situ (infographie M.-L. Maraval).

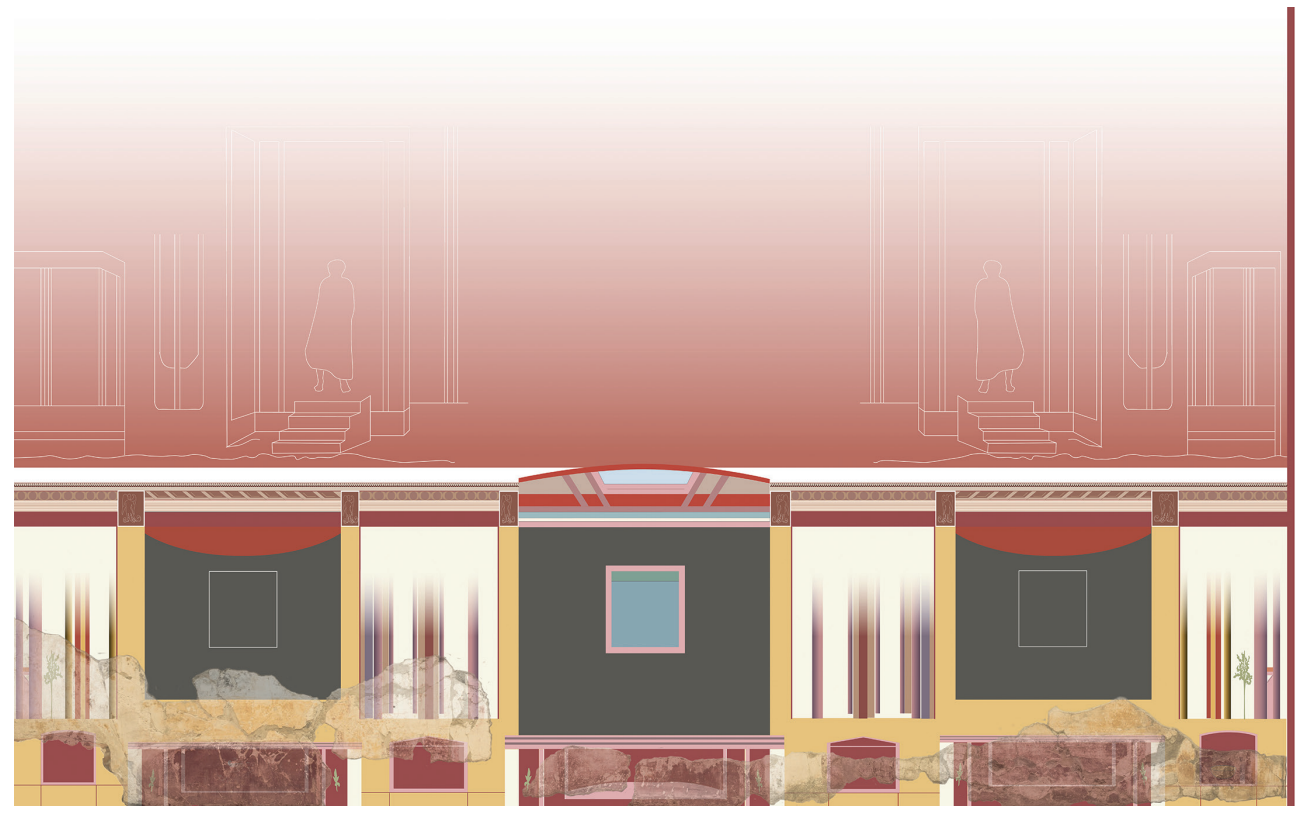

2. Maison de Neptune et Amphitrite, atrium 10, mur nord, restitution (infographie M.-L. Maraval). 


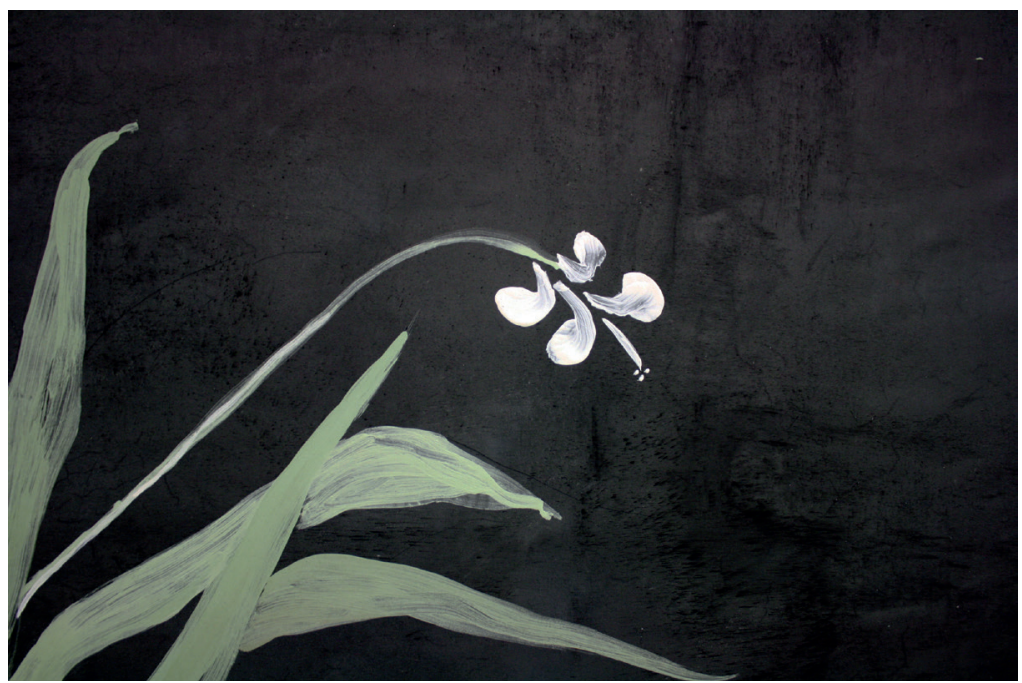

1. Détail du soubassement de la fresque expérimentale réalisée pour l'exposition L'Empire de la Couleur, de Pompéi au Sud des Gaules, Musée Saint-Raymond, Musée des Antiques de Toulouse, 2014-2015. Irrégularité du fond pourtant traité en aplat (fusées, taches...) et transparence de la touche picturale qui laisse voir le fond noir créant de nombreuses nuances de couleurs.

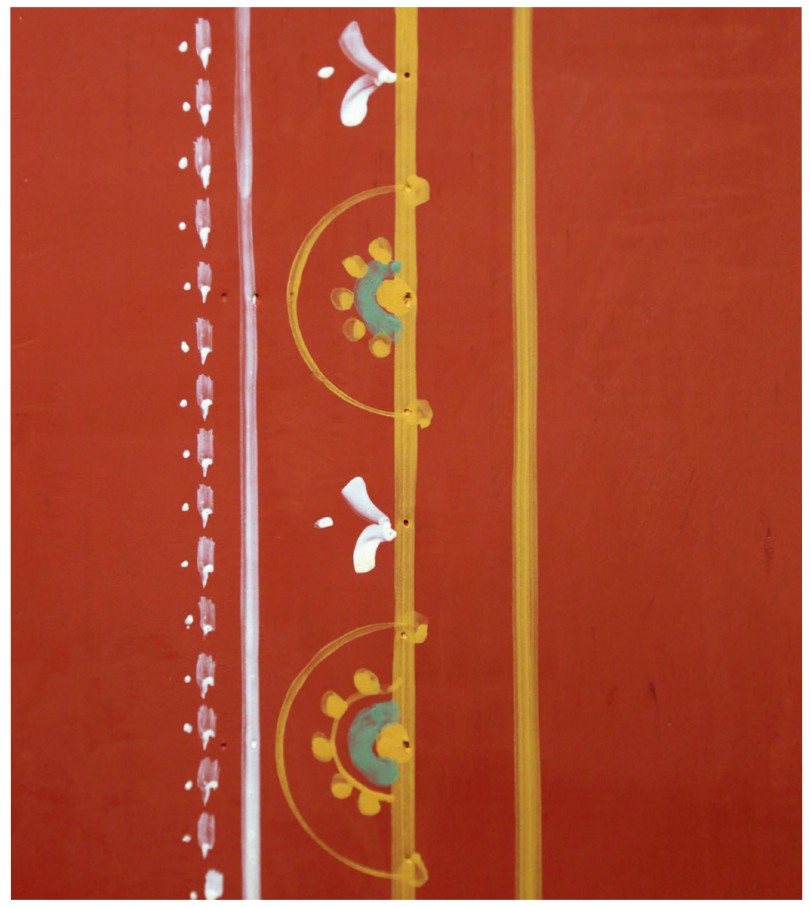

2. Détail d'un panneau

en zone médiane de la fresque expérimentale réalisée pour l'Exposition L'Empire de la Couleur, de Pompéi au Sud des Gaules, Musée Saint-Raymond, Musée des Antiques de Toulouse, 2014-2015. Bordures ajourées sur un fond rouge dont on distingue quelques irrégularités dans le fond ; les motifs répétitifs présentent aussi des irrégularités et des zones de transparence qui laissent voir le fond rouge. 


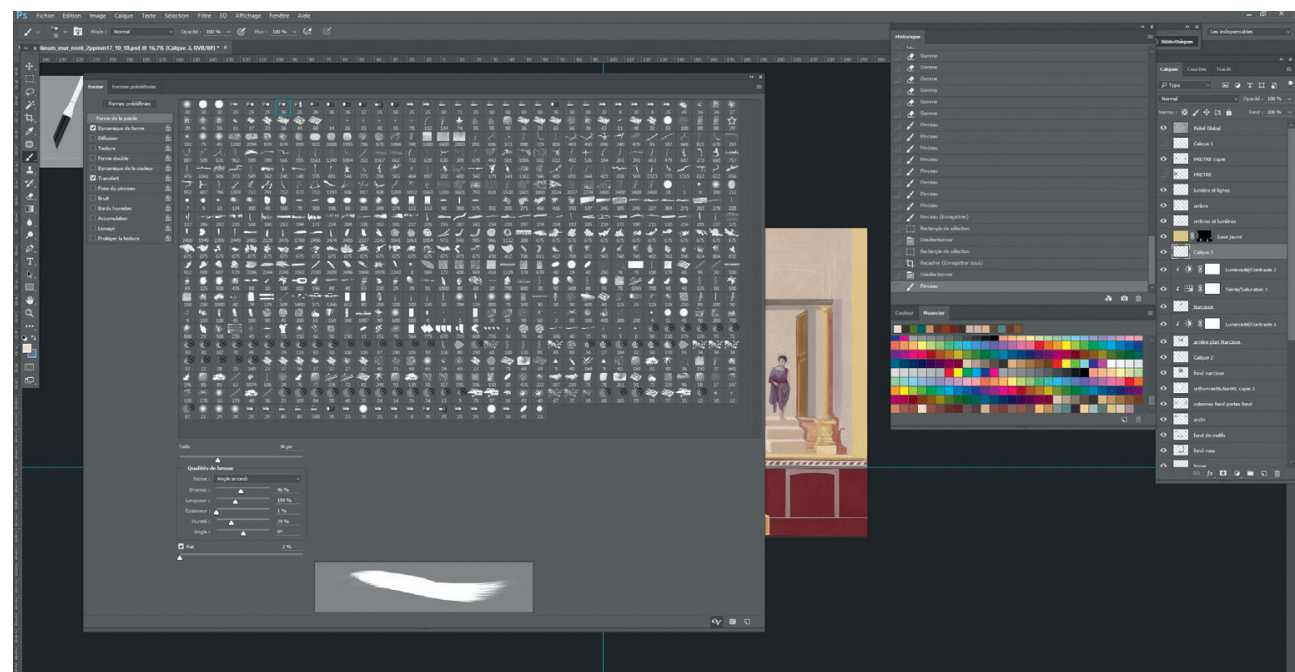

1. Fenêtre des brosses ouvertes dans le logiciel Adobe Photoshop, permettant de voir leur variété de formes et une partie des réglages possibles ; une brosse dynamique est sélectionnée (sa position est visible en haut à gauche). À l'arrière, une partie de la peinture virtuelle et à droite, sous l'historique, la palette des couleurs, puis les calques superposés.

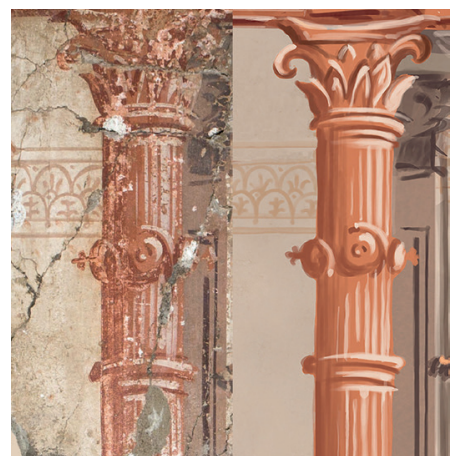

2. « Restauration virtuelle » d'une colonne du triclinium de la Maison de Neptune et Amphitrite à Herculanum : la brosse utilisée pour les touches blanches a permis de garder en transparence le fond lorsqu'elle est appliquée rapidement et au contraire de créer des effets d'accumulation correspondant au levé du pinceau qui produit une zone plus opaque (cf. pl. IX.2).

3. Détail de motifs répétitifs réalisés à main levée sur un fond rouge présentant des irrégularités. Peinture virtuelle du mur sud du triclinium 7 de la Maison de Neptune et Amphitrite à Herculanum.

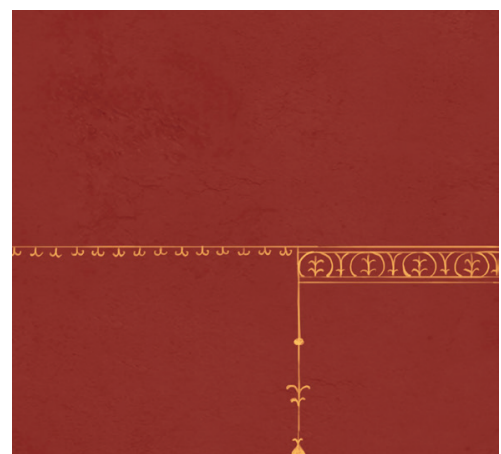




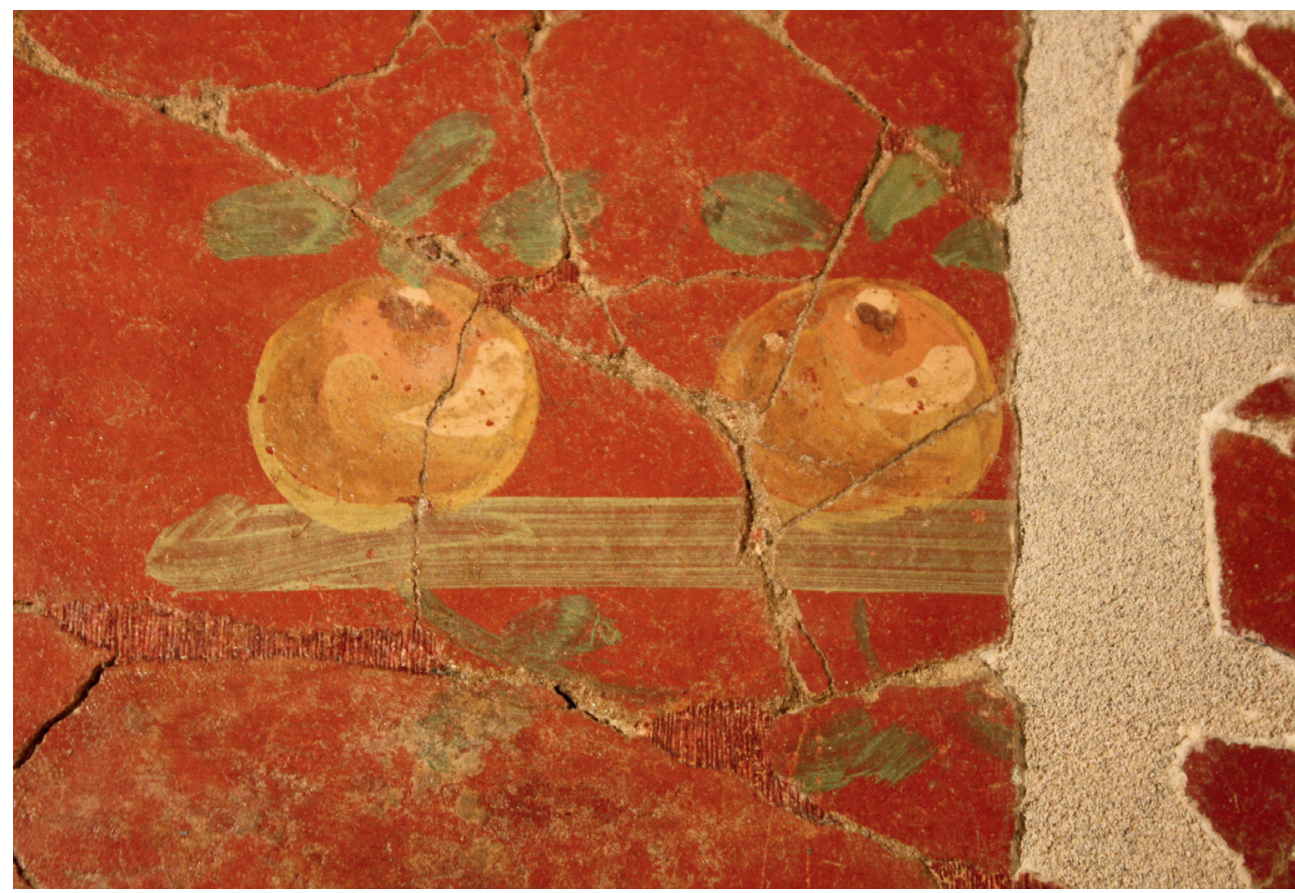

1. Détail d'une peinture provenant du Clos de la Lombarde, Musée de Narbonne. Fruits peints sur fond rouge réalisés à partir de plusieurs couleurs mélangées en amont et juxtaposées de façon à créer un dégradé afin de produire leur rendu sphérique ; le vert des feuilles et du plan laisse voir les effets du pinceau et transparaître la couleur de fond.

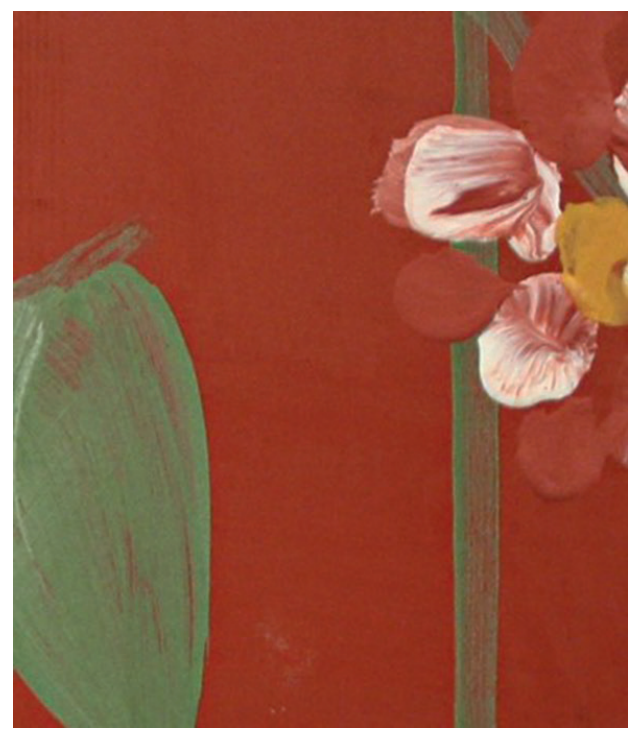

2. Détail d'un essai de peinture

à fresque réalisé en amont de la peinture exposée au Musée Saint-Raymond : le mélange des couleurs pures appliquées directement sur le support pour la réalisation des pétales roses crée un effet pictural très éloigné de celui d'une peinture romaine, contrairement au feuillage vert, où la couleur appliquée uniformément laisse transparaître le fond créant ainsi des variations de tons. 

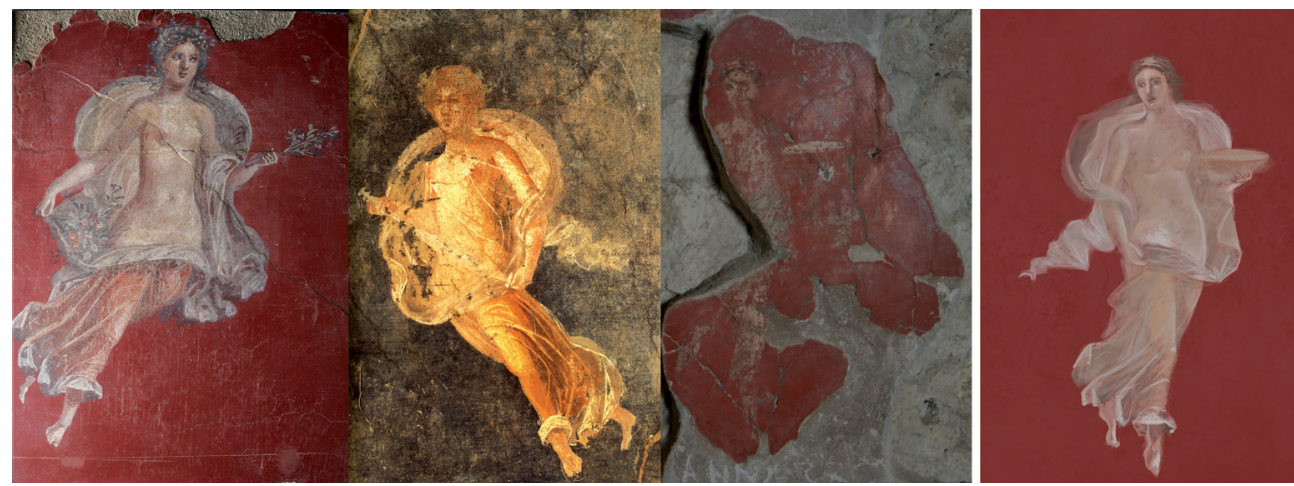

1. Restauration numérique d'une figure volante (à droite) à partir (de gauche à droite) d'une figure provenant de la même salle mais détachée et conservée au MANN ( $\mathrm{n}^{\circ}$ inv. 8835), d'une autre figure volante, sur fond noir, provenant de la maison de Holconius Rufus à Pompéi, et de ses vestiges conservés in situ et très effacés.

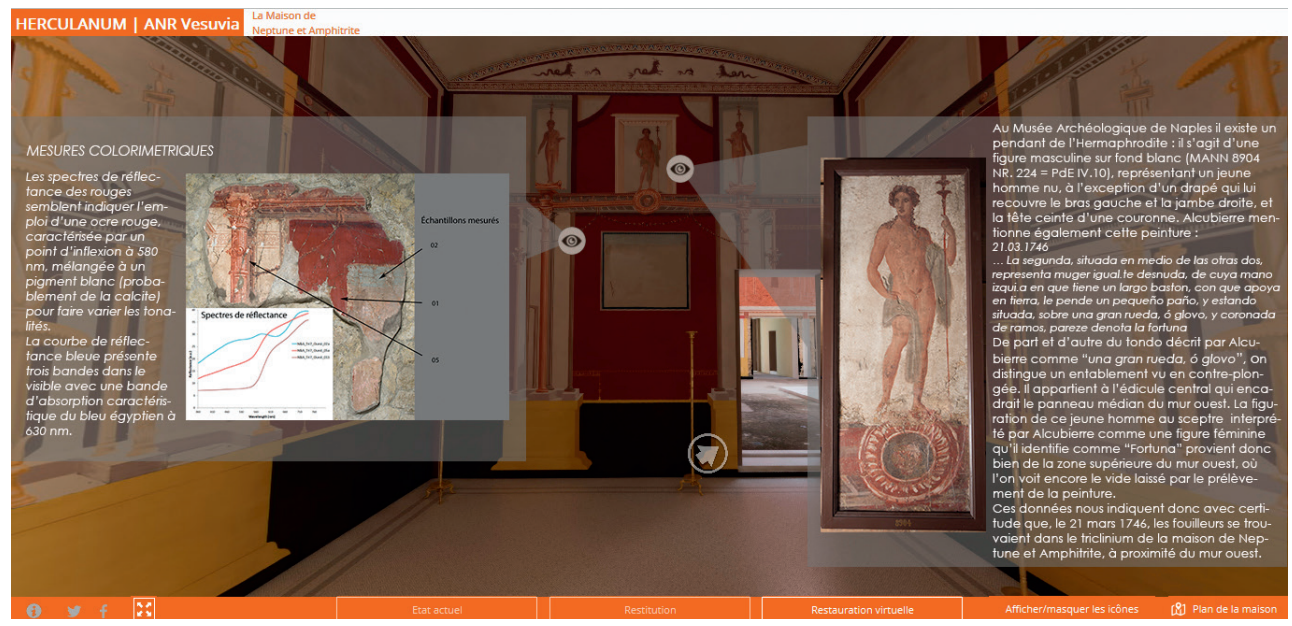

2. Vue du modèle 3D du triclinium 7 de la maison de Neptune et Amphitrite à Herculanum, réalisé par Archéovision-Archéotransfert, avec sa restauration numérique et deux zones cliquables ouvertes pour accéder à des informations complémentaires (ici des mesures colorimétriques et des données sur un panneau conservé au MANN et le contexte de sa découverte) ; au centre un panneau suggéré car aucun indice ne permet d'identifier la scène qui y était représentée. 